Supplement of Solid Earth, 9, 505-529, 2018

https://doi.org/10.5194/se-9-505-2018-supplement

C Author(s) 2018. This work is distributed under

the Creative Commons Attribution 4.0 License.

(c) (1)

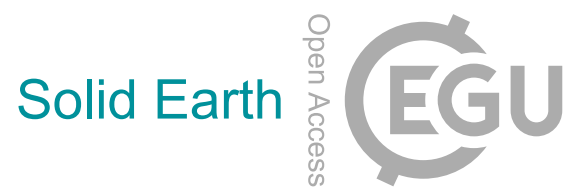

Supplement of

\title{
High stresses stored in fault zones: example of the Nojima fault (Japan)
}

Anne-Marie Boullier et al.

Correspondence to: Anne-Marie Boullier (anne-marie.boullier@univ-grenoble-alpes.fr)

The copyright of individual parts of the supplement might differ from the CC BY 4.0 License. 


\section{Supplementary material}

a - Alignements of tiny fluid inclusions
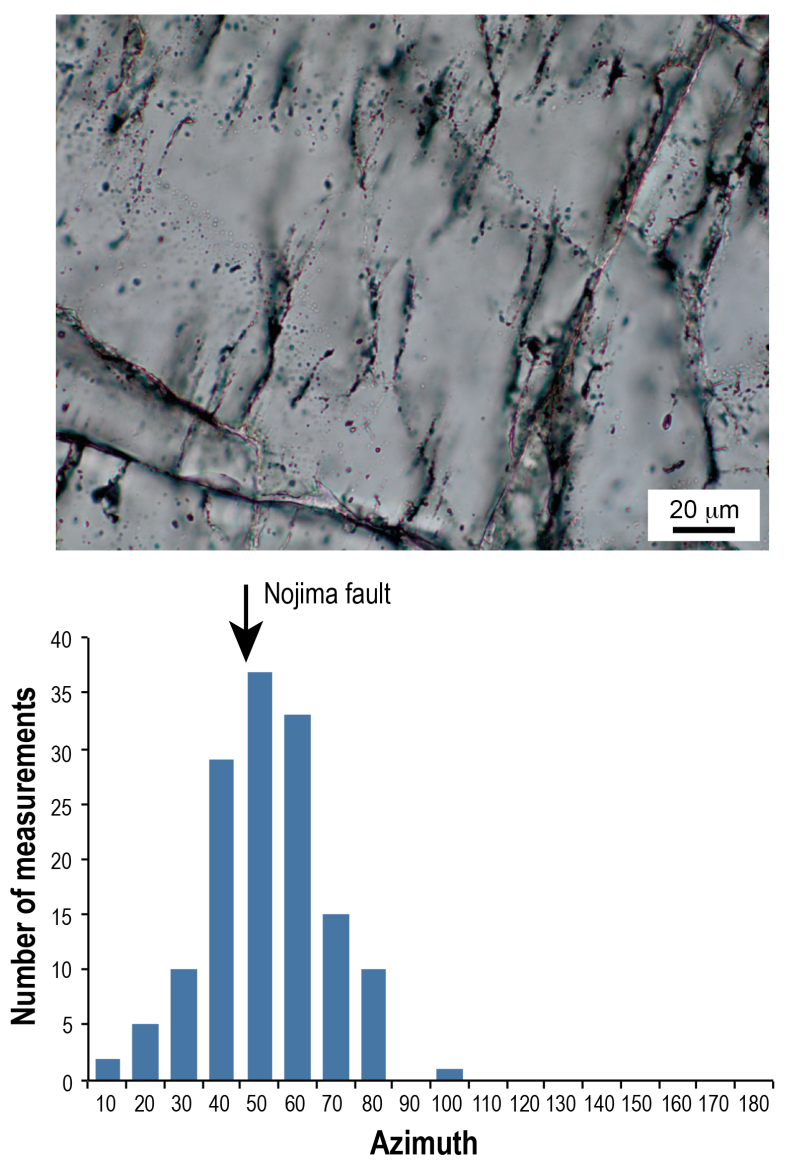

b - Kink-bands in biotites

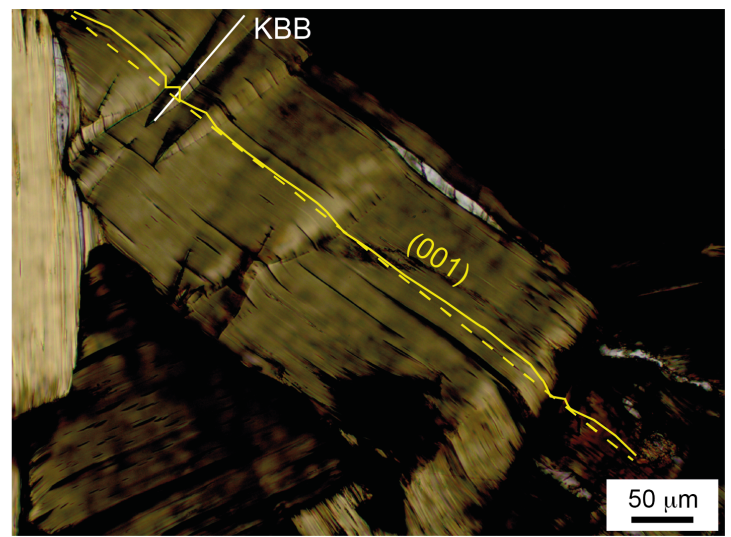

$\downarrow^{\text {Nojima fault }}$

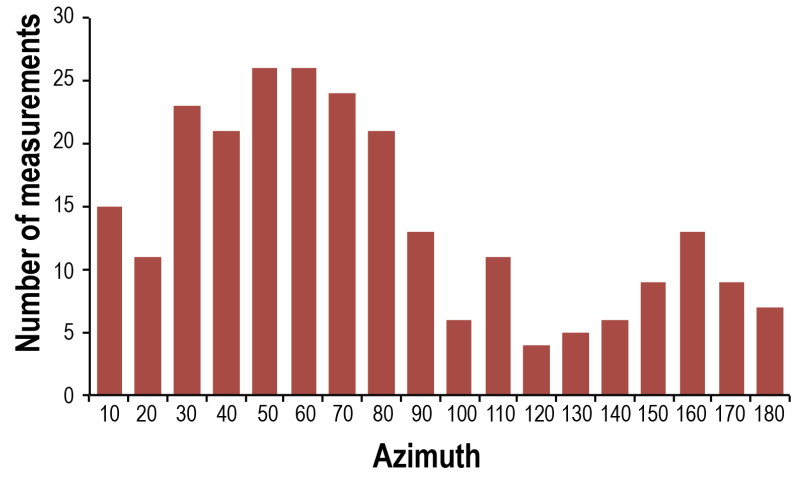

Figure S1 - Microphotographs and orientation histograms of (a) alignments of tiny fluid inclusions and (b) kinks (white line) in biotites in the NOJ220 sample. The yellow continuous line represents the trace of shortened (001) cleavage and the dashed line the final length of this cleavage allowing the strain measurement. 

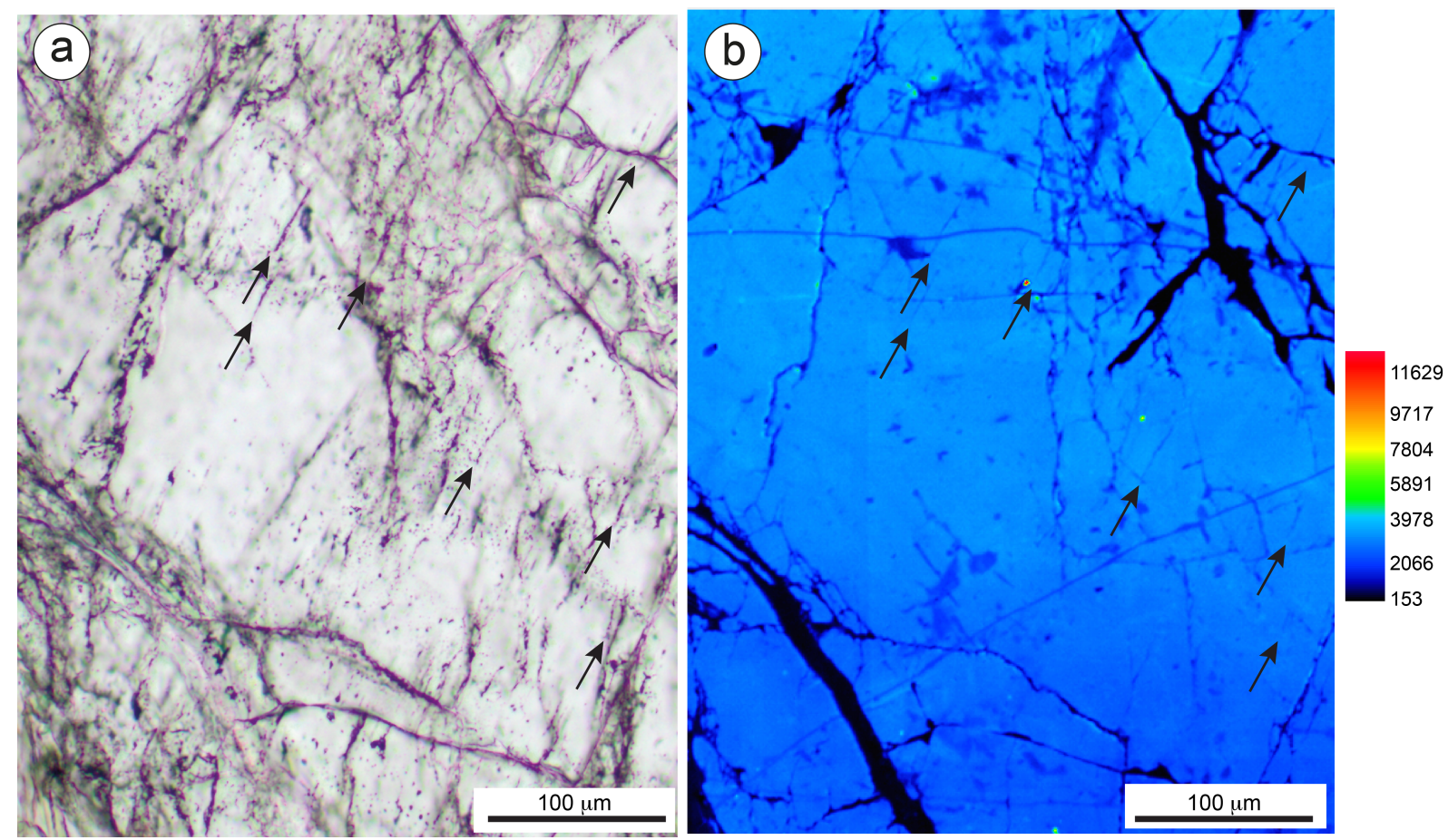

Figure S2 - Plane polarized microphotograph (a) and SEM-cathodoluminescence image (b) of the quartz studied by EBSD (Figure 4). The SEM-cathodoluminescence image was acquired on the Electron Probe X-ray Microanalyzer (EMPA) JEOL JX-8230 equiped with panchromatic cathodoluminescence system (ISTerre, Grenoble), with $15 \mathrm{kV}$ acceleration voltage, $10 \mathrm{nA}$ probe current, $1 \mu \mathrm{m}$ step size and $50 \mathrm{~ms}$ dwell time. Whereas the plane polarized micrograph integrates several tens $\mu \mathrm{m}$ in depth, cathodoluminescence image provides informations on the very near surface of the polished thin section. Therefore, microsructures visible on a may be not visible in $b$. The alignments of tiny fluid inclusions (black arrows) have a dark blue signature similar to quartz located near laumontite-filled microfractures suggesting both microstructures were healed by the same fluid. Note that some straight and thin microfractures (roughly E-W on the SEM cathodoluminescence image) have also the same dark blue signature but are not visible on the microphotograph in a) probably because they did not trapped fluid inclusions. 

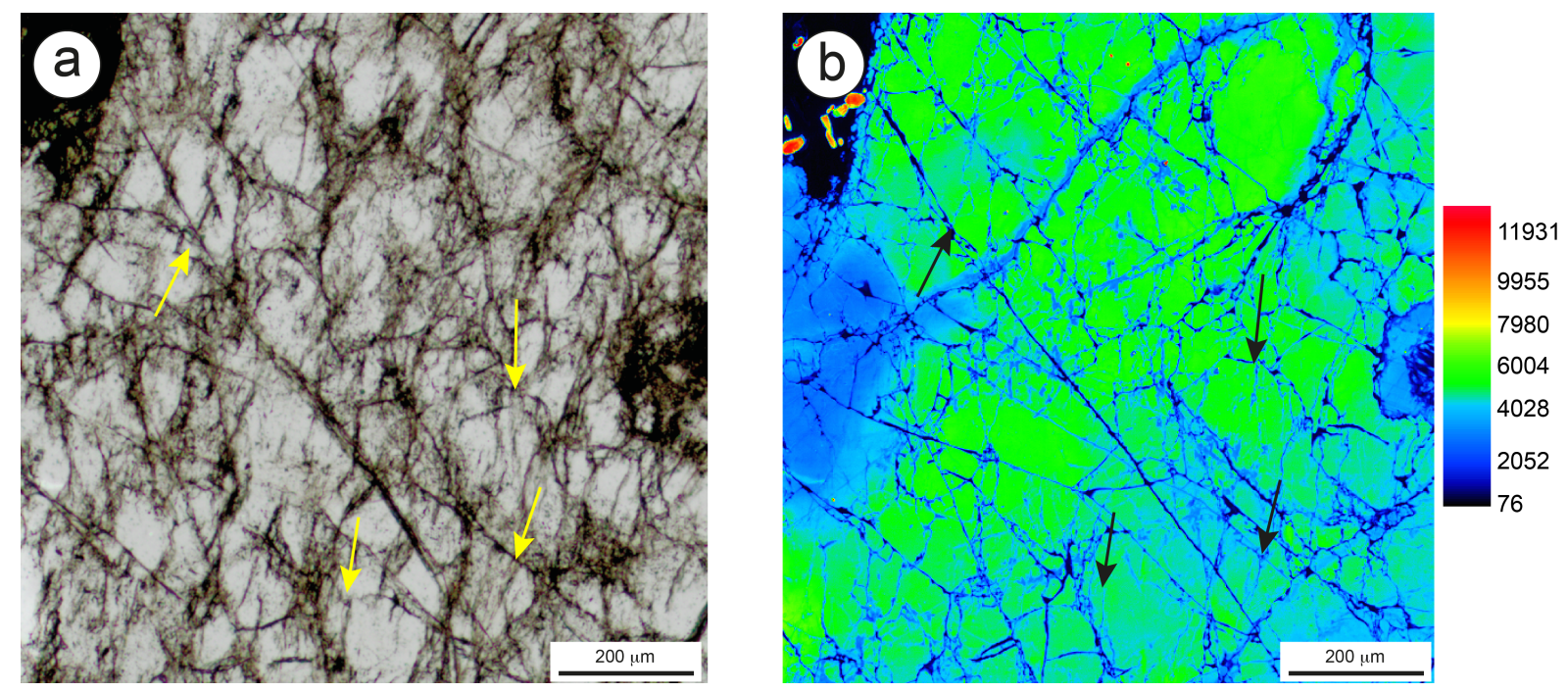

Figure S3 - Plane polarized microphotograph (a) and SEM-cathodoluminescence image (b) of the quartz studied by EBSD (Figure 7 and 8). The SEM-cathodoluminescence image was acquired on the Electron Probe X-ray Microanalyzer (EMPA) JEOL JX-8230 equiped with panchromatic cathodoluminescence system (ISTerre, Grenoble), with $15 \mathrm{kV}$ acceleration voltage, $20 \mathrm{nA}$ probe current, $1 \mu \mathrm{m}$ step size and $50 \mathrm{~ms}$ dwell time. Whereas the plane polarized micrograph integrates several tens $\mu \mathrm{m}$ in depth, cathodoluminescence image provides informations on the very near surface of the polished thin section. Therefore, microsructures visible on a may be not visible in $b$. The alignments of tiny fluid inclusions (yellow arrows on a, black arrows in b) have a dark blue signature similar to quartz located near laumontite-filled microfractures suggesting both microstructures were healed by the same fluid. 

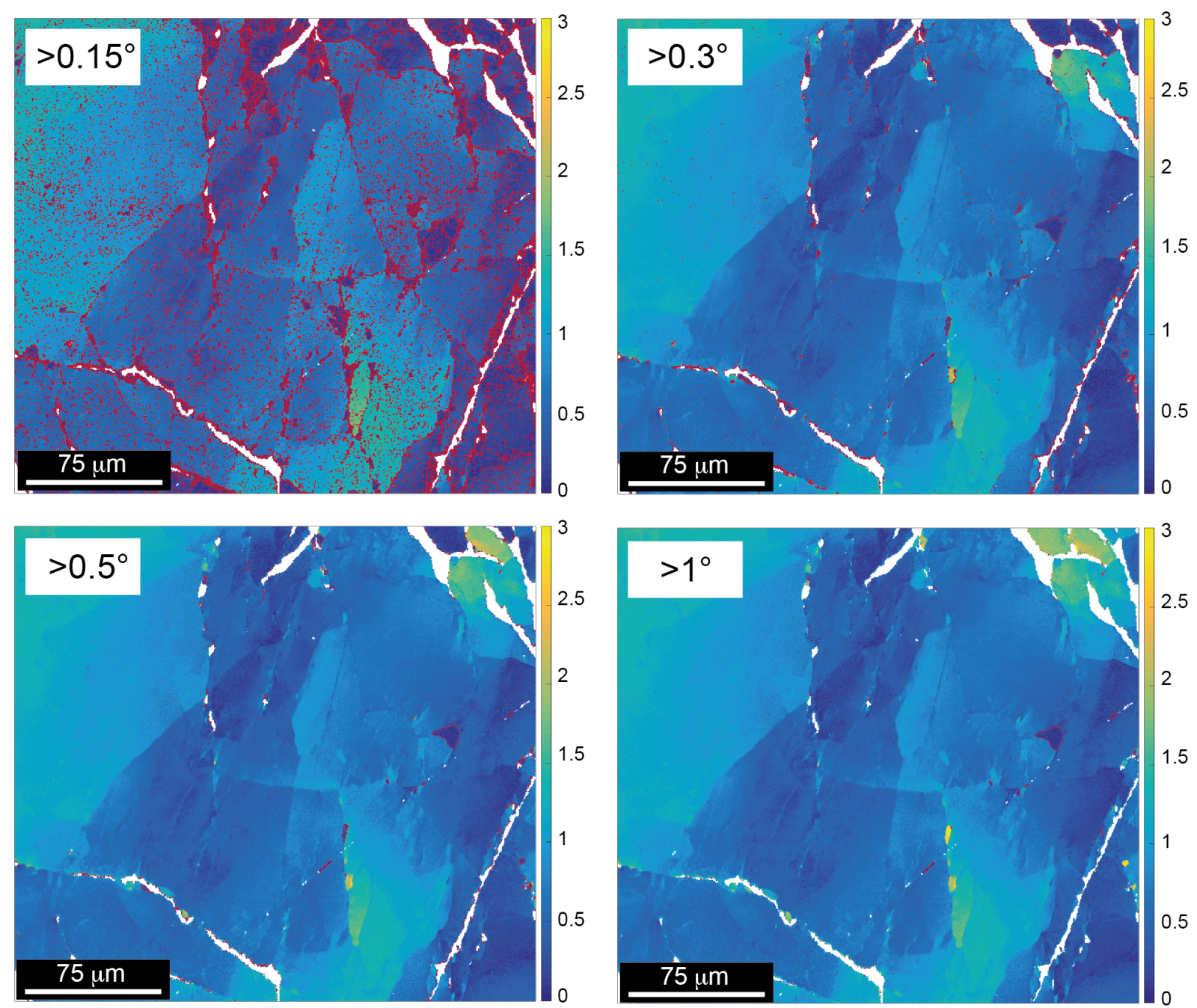

Figure S4 - Misorientation domains in the area studied in detail (Figure 10). The vertical or horizontal limits between pixels showing a misorientation greater than $0.15^{\circ}(\mathrm{a}), 0.3^{\circ}(\mathrm{b}), 0.5^{\circ}(\mathrm{c})$ and $1^{\circ}(\mathrm{d})$ are coloured in red. 

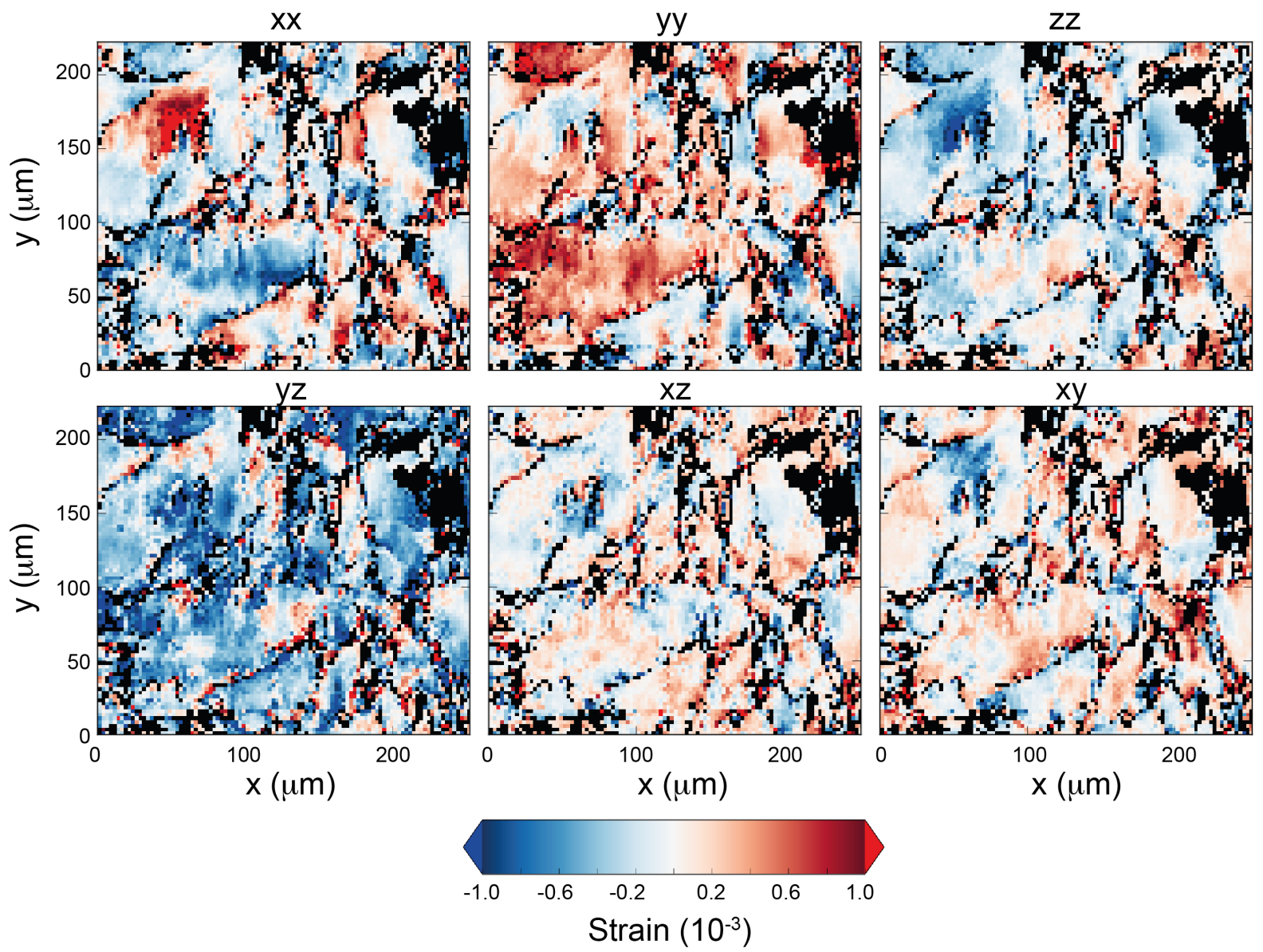

Figure S5 - X-ray Laue microdiffraction : maps of the six components of the deviatoric strain tensor, in the sample reference frame. Black points were filtered out based on the criteria described in Figure A2d. 
Section S1: Mathematical transformations applied to the EBSD data in order to pass the orientation matrices and map points positions into the LaueTools sample reference frame

These transformations were used to generate figures 9 and $11 \mathrm{~b}$.

The x,y map positions in the LT "map" sample reference frame (given in figure 2a) were calculated using :

x_map_LT $=x \_m a p \_E B S D$

ymap_LT $=$-ymap_EBSD

A first orientation matrix t_g_matrix was calculated from the Bunge Euler angles (e.g. Bunge, 1982) of the Oxford Instruments *.ctf file (components: $0=$ Euler1, $1=$ Euler2, $2=$ Euler3), using the formula given by R. Quey at page 10 in the Orilib Manual https://sourceforge.net/projects/orilib/files/orilib/manual\%201.1.1\%20\%28for\%20orilib\%201.1\%29/orilib-man1.1.1.pdf/download

phi1 $=$ Euler3_rad[0]

PHI = Euler3_rad[1]

phi2 $=$ Euler3_rad[2]

$\mathrm{g} 11=\cos ($ phi 1$) * \cos ($ phi2 $)-\sin ($ phi 1$) * \sin ($ phi 2$) * \cos (\mathrm{PHI})$

$\mathrm{g} 12=\sin (\mathrm{phi} 1) * \cos (\mathrm{phi} 2)+\cos ($ phi 1$) * \sin (\mathrm{phi} 2) * \cos (\mathrm{PHI})$

$\mathrm{g} 13=\sin (\mathrm{phi} 2) * \sin (\mathrm{PHI})$

$\mathrm{g} 21=-\cos ($ phi1 $) * \sin ($ phi2 $)-\sin ($ phi1 $) * \cos ($ phi2 $) * \cos (\mathrm{PHI})$

$\mathrm{g} 22=-\sin ($ phi1 $) * \sin ($ phi2 $)+\cos ($ phi1 $) * \cos ($ phi2 $) * \cos (\mathrm{PHI})$

$\mathrm{g} 23=\cos (\mathrm{phi} 2) * \sin (\mathrm{PHI})$

$\mathrm{g} 31=\sin ($ phi1 $) * \sin (\mathrm{PHI})$

$\mathrm{g} 32=-\cos ($ phi1 $) * \sin (\mathrm{PHI})$

$\mathrm{g} 33=\cos (\mathrm{PHI})$

g_matrix $=\operatorname{array}([[\mathrm{g} 11, \mathrm{~g} 12, \mathrm{~g} 13],[\mathrm{g} 21, \mathrm{~g} 22, \mathrm{~g} 23],[\mathrm{g} 31, \mathrm{~g} 32, \mathrm{~g} 33]])$

t_g_matrix $=$ g_matrix.transpose ()

Transformations were then applied to t_g_matrix using the criterion that for site 2, the final EBSD matrix should approximately match the microLaue matrix, following the three steps described below.

A first cartesian reciprocal unit cell (vectors $\mathrm{a} 0 *$, b $0 *, \mathrm{c} 0 *$ ) was created assuming that $\mathrm{t} \_\mathrm{g}$ _matrix gives the coordinates of $-\mathrm{a} 0 *,-\mathrm{b} 0 *, \mathrm{c} 0 *$ as columns on the $\mathrm{x}, \mathrm{y}, \mathrm{z}$ axes of the "orientation" EBSD sample reference frame (using the Matlab notation where mat[:,0] is the first column of the $3 \times 3$ mat matrix).

Ustarsample[:,0] = - t_g_matrix[:,0]

Ustarsample[:,1] = - t_g_matrix[:,1]

Ustarsample[:,2] = t_g_matrix[:,2]

Then a hexagonal reciprocal unit cell (vectors $\mathrm{a}^{*}, \mathrm{~b}^{*}, \mathrm{c}^{*}$ ) (for describing the trigonal quartz unit cell) matstarsample $3 \times 3$ was built using :

matstarsample $3 \times 3=\operatorname{dot}($ Ustarsample, Bstar)

where the reciprocal metric tensor Bstar describes the distorted shape of the reciprocal unit cell.

Bstar $=$

[[2.35029623e-01 1.17514812e-01 -2.97580061e-17]

$\left[\begin{array}{lll}0.00000000 \mathrm{e}+00 & 2.03541624 \mathrm{e}-01 & -1.13309289 \mathrm{e}-17\end{array}\right]$

$\left[\begin{array}{lll}0.00000000 \mathrm{e}+00 & 0.00000000 \mathrm{e}+00 & 1.85048113 \mathrm{e}-01\end{array}\right]$

This means that $\mathrm{a} 0 *$ is along $\mathrm{a}^{*}$ (direction + sense), i.e. $\mathrm{a}^{*}$ is the preferred direction for the orthonormalization process when building the reciprocal cartesian crystal frame from the reciprocal hexagonal unit cell. 
A 180 degrees rotation of the matrix around the z-axis was then applied in order to obtain the coordinates of the reciprocal crystal vectors in the "orientation" microLaue sample reference frame described in figure 2a (rotated by 180 degrees around $\mathrm{z}$ with respect to the "orientation" EBSD sample reference frame).

matrot $=$

[[-1. - -0. 0.]

[0. - 1.0.$]$

[0. 0.1.$]]$

matstarsample3 3 3_rotated $=\operatorname{dot}($ matrot, matstarsample $3 \times 3)$

At this stage, due to the arbitrary way of naming the $a^{*}, b^{*}, c^{*}$ reciprocal vectors when indexing, the matrix matstarsample3x3_rotated recalculated from EBSD may still differ from the microLaue matrix by a rotation of 120 degrees around $\mathrm{c}$ and/or an inversion of $\mathrm{c}$.

An "Euler angles minimization" process was therefore applied to get a single matrix for each crystallite.

From a given set of $\left(a^{*}, b^{*}, c^{*}\right)$ vectors, 6 equivalent matrices $\left(a^{*}\right.$ new, $b^{*}$ new, $\mathrm{c}^{*}$ new) can be built :

$\left(a^{*}, b^{*}, c^{*}\right),\left(-a^{*}+b^{*},-a^{*}, c^{*}\right),\left(-b^{*}, a^{*}-b^{*}, c^{*}\right)(120$ degrees rotations around $c)$

$\left(b^{*}, a^{*},-c^{*}\right),\left(a^{*}-b^{*},-b^{*},-c^{*}\right),\left(-a^{*}, b^{*}-a^{*},-c^{*}\right)\left(180\right.$ degrees rotation around $a^{*}+b^{*}$ then 120 degrees rotations around c)

The criteria used to choose between these 6 matrices was the following :

- the z-coordinate of $\mathrm{c}^{*}$ new should be positive (choice between $+\mathrm{c}^{*}$ and $-\mathrm{c}^{*}$ )

- the $\mathrm{x}$-coordinate of $\mathrm{a}^{*}$ new should be maximal (choice between the three matrices with the same $\mathrm{c}^{*}$ new)

This minimization process is important to ensure that all the map points belonging to closely oriented crystallites end up with similar matrices: this allows plotting usable histograms of orientation matrix components, and using these histograms to define thresholds on the orientation matrix components to class the map points according to the crystallite they belong to, and calculate the average orientation matrix of a given crystallite.

\section{Reference:}

Bunge, H.-J.: Texture analysis in materials science - Mathematical methods, Butterworths, London, UK, 1982. 


\section{Section S2: Validity of the Von Mises stress used in the article.}

The trigonal symmetry of the quartz unit cell, and the particular values of the quartz elastic constants $\mathrm{C}_{\mathrm{ijk}}$, lead to a coupling between the deviatoric and the expansion parts of the strain and stress tensor.

As a first consequence, pure expansion strain $\left(\varepsilon_{11}=\varepsilon_{22}=\varepsilon_{33}=\varepsilon_{\text {dil }}\right)$ leads to some amount of (purely diagonal) deviatoric stress

$\left(\sigma_{33}-\sigma_{11}=\sigma_{33}-\sigma_{22}=24 \mathrm{MPa}\right.$ per $1 \mathrm{e}^{-3}$ of $\left.\varepsilon_{\text {dil }}\right)$

As a second consequence, pure deviatoric strain leads to some amount of expansion stress, as illustrated for a few cases in Table S1.

As a third consequence, as also illustrated in table 1, the "pseudo von Mises" factor calculated here from pure deviatoric strain may deviate from the real von Mises factor, which depends on expansion strain.

The "pseudo von Mises stress" depends on $\varepsilon_{\text {dil }}$ via the two terms $\left(\sigma_{11}-\sigma_{33}\right)$ and $\left(\sigma_{22}-\sigma_{33}\right)$, which vary linearly with $\varepsilon_{\text {dil }}$, with factors $2 \mathrm{C}_{11}-2 \mathrm{C}_{13}+\mathrm{C}_{12}-\mathrm{C}_{33}=+51.23 \mathrm{GPa}$ and $\mathrm{C}_{12}+\mathrm{C}_{11}-\mathrm{C}_{33}-\mathrm{C}_{13}=-23.68 \mathrm{GPa}$

$\sigma_{1}-\sigma_{3}=\mathrm{C}_{11} *\left(\varepsilon_{1}+\varepsilon_{3}\right)-\mathrm{C}_{13} *\left(\varepsilon_{1}+\varepsilon_{2}\right)+\mathrm{C}_{12} * \varepsilon_{2}-\mathrm{C}_{22} * \varepsilon_{3}+\mathrm{C}_{14} * \varepsilon_{4}$

$\sigma_{2}-\sigma_{3}=C_{12} * \varepsilon_{1}+C_{11} * \varepsilon_{2}-C_{33} * \varepsilon_{3}+C_{13} *\left(-\varepsilon_{1}-\varepsilon_{2}+\varepsilon_{3}\right)+C_{24} * \varepsilon_{4}$

(with $\varepsilon_{1}=\varepsilon_{11}$ and $\varepsilon_{4}=2 * \varepsilon_{23}$ )

When deviatoric strain $\varepsilon_{\text {dev }}$ (the only part measured here) is converted into stress, the resulting "stress_from_ $\varepsilon_{\text {dev }}$ is a mixed incomplete quantity, that contains both deviatoric and expansion components, but none of them complete. For simplicity, we use the term "Von Mises stress" in the paper as a shortcut for "pseudo Von Mises stress calculated from deviatoric strain".

\begin{tabular}{|c|c|c|c|c|c|}
\hline$\varepsilon_{\mathrm{dil}}$ & $\varepsilon_{\mathrm{dev}}$ & $\sigma$ & $\sigma_{\text {dil }}$ & von Mises & $\mathrm{d}(\mathrm{vM}) / \mathrm{vM}$ \\
\hline 1111000 & 00000000 & 10510529000 & & 24 & \\
\hline $\begin{array}{l}0 \\
1 \\
-1\end{array}$ & 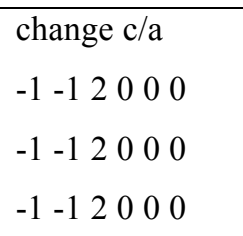 & $\begin{array}{l}-70-70187000 \\
3636316000 \\
-175-17558000\end{array}$ & 16 & $\begin{array}{l}257 \\
281 \\
233\end{array}$ & $\begin{array}{l}-8.4 \\
+10.1\end{array}$ \\
\hline $\begin{array}{l}0 \\
1 \\
-1\end{array}$ & 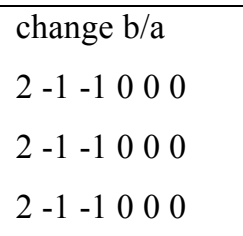 & $\begin{array}{l}155-85-94-5400 \\
2602136-5400 \\
49-190-223-5400\end{array}$ & -8 & $\begin{array}{l}261 \\
251 \\
274\end{array}$ & $\begin{array}{l}+4.3 \\
-4.5\end{array}$ \\
\hline $\begin{array}{l}0 \\
1 \\
-1\end{array}$ & $\begin{array}{l}\text { change angle } \beta \\
\begin{array}{lllllll}0 & 0 & 0 & 1 & 0 & 0 \\
0 & 0 & 0 & 1 & 0 & 0 \\
0 & 0 & 0 & 1 & 0 & 0\end{array}\end{array}$ & $\begin{array}{l}-36360116000 \\
6914212911600 \\
-142-69-12911600\end{array}$ & 0 & $\begin{array}{l}211 \\
212 \\
212\end{array}$ & $\begin{array}{l}-0.6 \\
-0.6\end{array}$ \\
\hline $\begin{array}{l}0 \\
1\end{array}$ & $\begin{array}{l}\text { change angle } \alpha \\
0000010 \\
0000010\end{array}$ & $\begin{array}{l}0000116-36 \\
1051051290116-36\end{array}$ & 0 & $\begin{array}{l}211 \\
212\end{array}$ & -0.6 \\
\hline $\begin{array}{l}0 \\
1\end{array}$ & $\begin{array}{l}\text { change angle } \gamma \\
0000001 \\
0000001\end{array}$ & $\begin{array}{l}000036-80 \\
105105129036-80\end{array}$ & 0 & $\begin{array}{l}152 \\
154\end{array}$ & -1.2 \\
\hline
\end{tabular}


Table S1 : Examples of errors induced by $+/-10^{-3}$ of expansion strain when using the "pseudo von Mises stress" (calculated from deviatoric strain only) instead of the full von Mises stress, for various types of deviatoric strain with components of the order of $10^{-3}$. The largest error is $24 \mathrm{MPa}$, obtained either for zero deviatoric strain or for a change in the c/a ratio. The error is negligible in the case of pure shear strain. Units : strain $\varepsilon_{\text {dev }}: 10^{-3}$; stress $\sigma: M P a$; error on von Mises stress $d(v M) / v M$ : percent. The stress and strain components are expressed in the cartesian frame attached to the quartz unit cell, and presented as a line in the order (11 22332313 12). The deviatoric stress induced by $+10^{-3}$ of pure expansion is $\left[\begin{array}{llllll}-8 & -8 & 16 & 0 & 0 & 0\end{array}\right]$. 